\title{
Über Ehre, Artuskritik und Dankbarkeit in Hartmanns Iwein
}

\author{
Karl Heinz Borck
}

Darf ein offenbar nicht mehr ganz taufrischer Herr königlichen Geblüts seine wohlversorgten Gäste im Stich lassen und sich am hellichten Tag mit seiner Gattin zu einem so oder so erquickenden Mittagsschläfchen zurückziehen? Bei Chrétien, der solches in seinem Löwenritterroman gleich zu Beginn bei der Schilderung des pfingstlichen Hoffestes von König Artus zu berichten weiß, reagieren die Ritter der Tafelrunde mit Verwunderung und offen geäußertem Verdruß. Hartmann scheint geahnt zu haben, daß es nötig sein würde, Artus gegen die manchmal etwas beckmesserisch anmutenden Attacken einer nachgeborenen Generation gelehrter Germanisten in Schutz zu nehmen, denn er ist sichtlich bestrebt, das Anstößige der Szene nicht so deutlich hervortreten zu lassen wie in seiner Quelle. Dennoch scheinen Artus und die Seinen auch in Hartmanns Erzählung immer wieder ins Zwielicht gerückt zu sein. Dies ist wenigstens die Meinung einer langen Reihe von Germanisten, die schon 1937 mit Ernst Scheunemann beginnt und bei der heute auch in der Mediaevistik feststellbaren Sensibilität für alles Gesellschaftskritische zweifellos als noch nicht abgeschlossen gelten darf. Daß sich gleich zweimal Londoner Germanisten, so schon 1961 Hugh Sacker und einundzwanzig Jahre später Silvia Ranawake, mit besonders wohlüberlegten Argumenten für die These ausgesprochen haben, das artushafte Aventiurerittertum sei in Hartmanns /wein durch eine betont ironische Darstellung bloßgestellt und der Kritik preisgegeben worden, läßt aufhorchen, denn wo sollte königliche Lebensart kompetentere Beurteiler finden als im Herzen des britischen Empire und am Stammsitz der in der Fleet Street beheimateten Regenbogenpresse? Doch Scherz beiseite, die von Sacker, Ranawake, Günther Schweikle und anderen vertretene Auffassung ist so gut begründet, daß sie sich wahrscheinlich schon durchgesetzt und als opinio communis etabliert hätte, stünde dem nicht der Prolog entgegen, den Hartmann, eher beiläufig wirkende Bemerkungen Chrétiens aufnehmend, zum wahren Musterfall eines die einschlägigen praecepta der zeitgenössischen Rhetorik befolgenden Redebeginns ausgebaut hat. Denn hier wird für künec Artûs den guoten genau das in Anspruch genommen, was ihm die aus der Romanhandlung erschlossene Kritik zu bestreiten scheint: die Verbindlichkeit eines die Zeiten überdauernden Vorbildes.

Das Problem, das sich in diesem unvermittelten Nebeneinander abzeichnet, harrt - 
wenn ich recht sehe - immer noch einer Lösung. Soweit die Verfechter einer ironisch-artuskritischen Deutung des Iwein es nicht vorziehen, über den sperrigen Prolog einfach hinwegzusehen, vermögen ihre ohnehin nur zögernd und ohne das nötige Selbstvertrauen unternommenen Harmonisierungsversuche nicht recht zu überzeugen. Ob es den folgenden Überlegungen, die dem gleichen Gegenstand gelten, in der hier versammelten Fachöffentlichkeit besser ergehen wird, bleibt abzuwarten. Daß ich sie Ihnen unter dem skurrilen Titel Über Ehre, Artusritik und Dankbarkeit in Hartmanns 'Iwein' vorlege, hat Gründe, die ich nun nicht mehr zu verhehlen brauche. Die scheinbar sinnlose Reihung disparater Begriffe sollte die Iwein-Kenner unter den Antipoden in moderate Spannung versetzen und allen Kollegen, die einer unzeitgemäßen Disziplin wie der Mediaevistik nur geringes Interesse entgegenbringen, mit der bereitwilligen Bestätigung ihrer Vorenwartung den Entschluß erleichtern, ihre Zeit andernorts und mit einer aufregenderen Beschäftigung hinzubringen. Nur Du, lieber Eric, mußt wohl oder übel hier bleiben und meinen Worten in der Haltung lauschen, die auch Hartmann seinen Hörern abverlangt: mit offnen Ohren und bewegtem Herzen.

Meine Überlegungen gehen von vier Prämissen aus:

1. Der Iwein-Prolog ruft nicht umsonst zur imitatio des Königs Artus auf. Wie die anderen epischen Werke Hartmanns ist auch der Iwein als Vorbilddichtung konzipiert. Im erzählten Geschehen des fiktiven maeres entialtet sich ein Sinn, mit dessen Wahrheitsanspruch konfrontiert sich die Hörer zum bilde nemen aufgefordert finden. In dieser Hinsicht entspricht Hartmanns dichterische Praxis präzise den literaturtheoretischen Erwägungen, die sein jüngerer Zeitgenosse Thomasin von Zerclaere im Winter $1215 / 16$ bei der Niederschrift des Welschen Gastes angestellt hat.

2. Hartmanns Intention muß lückenlos am mittelhochdeutschen Worlaut seines Iwein ablesbar sein. Vergleiche mit der Quelle haben lediglich heuristischen Wert, denn es ist nicht anzunehmen, daß Hartmanns Publikum mit dem Löwenritterroman Chrétiens vertraut war. Sinnstiftende Rückverweise auf die altfranzösische Vorlage waren unter diesen Umständen kaum ratsam.

3. Hartmanns Stammpublikum ist an einem jener großen Höfe zu suchen, die als Sitz des Lehnsherrn, Gerichtsort und Verwaltunigszentrum ständig Angehörige des alten Geblütsadels und der schon in sich stark differenzierten Ministerialität mit Vertretern des Klerikerstandes zu gemeinsamem Tun zusammenführten. Jede der genannten Gruppen partizipiert auf ihre Weise an der Ausübung von Herrschaft. 
4. Literarische Zeugnisse und rechtsgeschichtliche Fakten erlauben die Annahme, daß sich die Ministerialität bei ihrem Aufstieg in den Herrenstand mehr und mehr auch geistig in den Adel integriert hat. Um 1200 scheint das Selbstverständnis arrivierter Ministerialenfamilien nur noch durch ihre gesellschaftliche Position bestimmt zu sein und in allen wesentlichen Zügen dem der Dynastengeschlecher zu gleichen.

Erlauben Sie mir noch eine vorgreifende Bemerkung zum Geltungsanspruch der folgenden Überlegungen und den Hinweis auf ein methodisches Erfordernis, das nicht immer die Beachtung findet, die es nach meinem Darfürhalten verdient.

Der Versuch, die Frage zu erörtern, ob Artuslob und Artuskritik nicht doch in einer höheren Einheit aufgehoben sind, faßt nicht den ganzen twein ins Auge. Der Blick bleibt auf eine Sinnebene beschränkt. Alle weiteren Bedeutungsschichten des Romans werden bewußt ausgeblendet. Das besagt natürlich nicht, daß sie geleugnet oder für unwichtig gehalten würden. Methodisch hebt sich der hier gewähite Zugriff scharf von einer Verfahrensweise ab, die sich in der Abkehr von der textimmanenten Interpretation herausgebildet hat und auch in der jüngeren Hartmannforschung einer gewissen Beliebtheit erfreut. Dabei werden einzeine Textstellen aus dem Zusammenhang gelöst und zur Grundlage einer Gesamtdeutung gemacht, ob es sich nun bei den für allein maßgeblich erklärten Passagen um die Erzählerkommentare handelt, um Partien, die sich einem allegorischen Verständnis erschließen, oder solche, die der erwünschten sozialhistorischen Deutung besonders entgegenzukommen scheinen. Wer so verfährt, sieht allzu großzügig über die Ergebnisse hinweg, die seit dem richtungweisenden Erec-Aufsatz von Hugo Kuhn aus dem Jahre 1948 von der Strukturforschung vorgelegt worden sind. Im klassischen Artusroman führt der Weg des Protagonisten zu seiner wahren Identität über eine Reihe von Stationen, die sich zur Sinnstruktur des doppelten Kursus zusammenschließen. Welches Gewicht einer einzelnen Szene beigelegt werden darf, wieviel von der âventiure meine in dem hier erzählten Geschehen durchscheint, ergibt sich nicht zuletzt aus der Position, die ihr in der Symbolstruktur des Romanganzen zukommt. Dieser Einsicht ist auch bei einer knappen, nur skizzenhaften Textanalyse Rechnung zu tragen.

Bei Hartmann steht der erste Teil des Wwein ganz im Zeichen der êre. Kaum, daß die zu Artus' Pfingstfest in Karidol Versammelten mit der Nennung des Musterritters Gawein und des königlichen Truchsessen als Tafelrunde identifiziert worden sind, bricht der zuht/ôse Keii einen Streit vom Zaune. Ginover ist unvermutet an eine Gruppe von Rittern herangetreten, und nur einer von ihnen hat dies rechtzeitig 
bemerkt und der Königin die ihr gebührende Ehre erwiesen:

dô erzeicte aber Keî

sîn alte gewonheit:

im was des mannes êre leit,

und beruoft in drumbe sêre

und sprach im an sîn êre. (108-12)

Keies Ehrgeiz duldet nicht, daß anderen verdiente Anerkennung zuteil wird. Nur mit Mühe gelingt es der Königin, die gestörte Harmonie des Festes wiederherzustellen und so Kalogrenant Gelegenheit zu geben, eine bereits begonnene Erzählung wieder aufzugreifen. Was er zu berichten hat, macht verständlich, daß er sein Abenteuer zehn Jahre lang schamhaft verschwiegen hat und sich rückblickend einen tôren schilt, weil er das Geheimnis nun doch preisgegeben hat. Gewâfent nâch gewonheit auf der Suche nach ritterlicher Bewährung, sei er auf einer Burg zu Gast gewesen, deren Herr nie zuvor einen âventiure suchenden Ritter beherbergt habe. Tags darauf sei er einem furchterregenden Waldmenschen begegnet und von ihm auf den Weg zu einer Wunderquelle gebracht worden. Hier habe er, nach einigem Zögern der costume folgend, ein verheerendes Unwetter ausgelöst, sei vom herbeieilenden Herrn des Landes, König Ascalon, erst der triuwe-losigkeit, des Rechtsbruchs und mutwilliger Friedensstörung bezichtigt und dann ohne viel Federlesens aus dem Sattel gehoben worden. Die Rückkehr zum Artushof habe er als ein êrlôser man und bar der ritterlichen Attribute Roß und Rüstung antreten müssen. Soweit Kalogrenants maere. Iweins spontaner Entschluß, die Schmach seines Verwandten Kalogrenant zu rächen und so die Sippenehre wiederherzustellen, ruft sofort wieder den Spötter Keii auf den Plan. Erneut zeigt sich, daß die Solidarität der Tafelrunde im rücksichtslosen Ehrstreben einzelner ihre Grenze findet. Nicht nur für Keii. Als Artus schwört, in vierzehn Tagen mit seinem ganzen Gefolge am Wunderquell zu erscheinen, macht Iwein sich heimlich auf, um seinen Konkurrenten zuvorzukommen. Er stiehlt sich davon

[.. .] rehte als ein man

der êre mit listen

kunde gewinnen und vristen. (946-48)

Die Brunnenaventiuren Kalogrenants und Iweins verhalten sich zueinander wie Typus und Antitypus. Hartmanns Darstellung arbeitet die zwischen ihnen bestehende Analogie scharf heraus. Iwein muß die einzelnen Stationen des von seinem neven zurückgelegten Weges noch einmal rekapitulieren, ehe er aufbricht und sie Schritt für 
Schritt im Geiste Kalogrenants nachvollzieht. Nur erweist sich Iwein als der Erwählte, dessen manheit und saelde ihm zum Sieg verhelfen. Als Ascalon, tödlich verwundet, die Flucht ergreift und Iwein Gefahr läuft, ohne jeden Beweis für seinen Erfolg dem ehrvernichtenden spot Keies ausgesetzt zu sein, jagt er hinter dem Fliehenden her, erreicht inn schon innerhalb der Burgbefestigungen und versetzt dem Wehrlosen noch einen letzten Schwerthieb, ehe Fallgitter niedersausen und den Sieger zum Gefangenen machen. Es erübrigt sich, die heißumstrittene Frage zu erörtern, ob Hartmann mit der lapidaren Feststellung her Iwein jaget in âne zuht (1056) die fehlende Selbstbeherrschung des Protagonisten moniert hat oder rein deskriptiv verfährt und sich mit der Mitteilung begnügt, Iwein habe die Verfolgung mit verhängten Zügeln aufgenommen. Auch die Suche nach einer Initialschuld Iweins kann unbedenklich eingestellt werden. Entscheidend ist allein der muot, die geistig-sittliche Grundhaltung, die in seinem gesamten Handeln sinnfällig zutage tritt. Bei der Verfolgung Ascalons bleibt erkennbar alles auf der Strecke, was dem bedingungslosen Ehrstreben des Artusritters Iwein und seiner Furcht vor Schmach und Schande entgegensteht. Hartmann hat den Geist solchen Artusrittertums in einer der Schiüsselszenen des Romans, der Begegnung Kalogrenants mit dem waltôren, prägnant ins Wort gebracht. Auf die verwunderte Frage des Hirten 'âventiure? waz ist daz?' (527) antwortet Kalogrenant mit einer Definition, die in der Forschung ohne zureichenden Grund als nur pädagogisch gemeint abqualifiziert worden ist:

daz wil ich dir bescheiden baz.

nû sich wie ich gewâfent bin:

ich heize ein riter und hân den sin

daz ich suochende rite

einen man der mit mir strîte, der gewâfent sî als ich.

daz prîset in, und sleht er mich:

gesige aber ich im an,

sô hât man mich vür einen man,

und wirde werder danne ich sî. (528-37)

Es ist kaum zu übersehen, daß Kalogrenants Definition, sein eigener Kampf gegen Ascalon und Iweins Brunnenaventiure völlig deckungsgleich sind. Der Sinn solcher âventiure erschöpft sich in kriegerischer Selbstbestätigung und in einem Ehrgewinn, der selbst die Tötung eines Mitmenschen zu rechtfertigen vermag, weil er an eine 
einzige Vorbedingung gebunden ist: daß er gewâfent sî als ich.

Kurt Ruh hat entschieden in Abrede gestellt, daß der von Kalogrenant formulierte Aventiurebegriff defizient sei und daß Iwein sich im Quellenabenteuer etwas zu Schulden kommen lasse. Wie Kalogrenant und später Artus selbst befolge er nur die Gesetze der costume und halte sich, auch darin, strikt an die Spielregeln des Artushofes, deren breit bezeugte Anerkennung moralische Zweifel ausschiieße. Gewiß werden Gawein und Keii, Kalogrenant und Iwein von Hartmann im ersten Teil des Romans als typische Artusritter dargestellt - aber bedeutet das bereits den Verzicht auf Kritik? Als literarisches Motiv verkörpern Artus und seine Tafeirunde das Ideal höfischen Rittertums. Wer dieses Ideal neu und tiefer begründen wolite, tat gut daran, den für notwendig erachteten Sinneswandel an einem Artusritter zu demonstrieren. Denn so eröffnete sich die Möglichkeit, die bisher gültigen Normen einer Korrektur zu unterziehen und gleichzeitig ihren Geltungsanspruch für das neue Ethos zu erhalten.

Auch der Versuch von Volker Mertens, die Frage nach Iweins Schuld und anderen 'artuskritischen' Elementen vor dem Hintergrund zeitgenössischer Rechtsbräuche zu beantworten, liefert keine entscheidenden Kriterien. Denn den sittlichen Maßstäben und Entwürfen Hartmanns waren durch das Gewohnheitsrecht sicher keine unübersteigbaren Grenzen gesetzt.

Unter diesen Umständen empfiehlt es sich, Hartmann selbst zu befragen, wo eben dies möglich ist. Das einzige literarische Werk, dessen Kenntnis der Iwein-Dichter bei seinem Publikum nachweislich voraussetzt, ist sein Erec. Und hier erfährt die âventiure in der Cadoc-Episode eine neue Sinngebung aus christichem Geist. Als Erec die beiden Riesen erreicht hat, die Cadoc gefangen mit sich führen, wird er gleich David vor dem Kampf gegen Goliath verhöhnt:

nû nim dir in ze mâge

und hilf im: dest im nôt genuoc. (5485f.)

Man ist versucht, mâc hier mit 'Bruder in Christo' oder 'Nächster' zu übersetzen. Erecs Sieg über die beiden vâlande, mit Gottes Hilfe und aus erbermde errungen, verleiht den höhnischen Worten eine neue Sinndimension, die in den Befreiungstaten der Schlu Baventiure Joie de la curt ihre Bestätigung findet. Zuvor hat Erec im zweiten Guivreizkampf einsehen gelernt, wohin es führt, wenn das Streben nach êre zum Selbstzweck wird, und seine Niederlage als gerecht akzeptiert.

Vor diesem Hintergrund ist auch die 'sprechende Situation' zu beurteilen, in die der zwischen den Fallgittern gefangene Iwein durch die mitleidslose Verfolgung Ascalons geraten ist. Iwein sitzt fest, kann weder vorwärts noch zurück, und ist auf die Hilfe 
Lunetes angewiesen, um wenigstens das nackte Leben retten zu können. Die Unzulänglichkeit der vom Streben nach êre beherrschten Ethik des Artushofs wird in seiner Erfahrung offenbar, aber Iwein vermag die darin liegende Forderung nach einer neuen Sittlichkeit nicht zu erkennen. Seine Sorge gilt nach wie vor der Furcht vor Keies spot und den fehlenden Beweisen für seinen Erfolg in der âventiure. E in e tiefgreifende Veränderung geht alierdings in lweins Leben vor: er erfährt die Allmacht der Minne. Aber die leidenschaftliche Liebe zu Ascalons Witwe, die ihn beim ersten Anblick Laudines ergreift, ergänzt und erweitert den Denkhorizont des Artusritters, ohne inn in Frage zu stellen. Um es in Hartmanns Sprache zu sagen: Iweins Gefangenschaft ist fortan eine doppelte.

Die paradoxe Lage des eingespertten und in Minnebande gefesselten Siegers wäre hoffnungslos, gäbe es nicht Lunete, der Iwein einst am Artushof als einziger unter allen Tafelrundern die schuldige Ehre erwiesen hat und die sich ihm daher zur Dankbarkeit verpflichtet fühlt. Lunete entzieht ihn nicht nur dem rächenden Zugriff der Leute Ascalons, sie schafft es sogar, Laudine davon zu überzeugen, daß niemand besser geeignet sei, ihrem Lande den unentbehrlichen Schutz zu gewähren, als der Ritter, der im Kampf mit Ascalon die Oberhand behalten und sich dadurch als tiurer erwiesen habe, denn

$$
\text { min herre ist tôt und er genesen (1970). }
$$

Die karge sittliche Logik des von Lunete erteilten und nach anfänglichem Widerstreben von Laudine akzeptierten râtes entspricht dem moralischen Gehalt der âventiure-Definition Kalogrenants. Diese Feststellung sollte davor bewahren, im Laudinebereich vorschnell den positiv bewerteten Gegenpol des Artushofes zu erblicken. Als die rasch getröstete Witwe an der Seite ihres zweiten Ehemannes Artus und seinem Gefolge Gasttreundschaft bieten darf, gilt ihr die Anwesenheit des Königs offensichtlich als besondere Auszeichnung. Mit Iweins manheit und êre erfahren nicht nur in ihren Augen auch das soziale Prestige Laudines und ihr problematischer EntschluB, Ascalons Bezwinger zu heiraten, eine Bestätigung durch die höchste Instanz. Nur in einer Hinsicht überschreitet Laudines Denken deutlich die Mentalität des Artuskreises. Sie weiß, daß ihr Land nur gedeihen kann, wenn es einen Schutzherrn hat, der ihm den Frieden erhält. Iweins Sieg über Keii scheint zu bestätigen, daß sie die richtige Wahl getroffen hat. Aber wie Iwein in der Ehe nur das Gefäß einer heiß ersehnten Liebesbeziehung erblickt, so bietet ihm der in der Rolle des Landesherrn geführte Kampf nur die wilikommene Gelegenheit, am Schandmaul Keii persönliche râche zu nehmen. Artushaftes Aventiurerittertum, wie Iwein es auch nach der Heirat 
mit Laudine und als herre dâ ze lande unverändert repräsentiert, erweist sich als blind für die Aufgaben der Friedenssicherung und Schutzgewährung. Das gilt auch für Gawein, den Musterritter des Artushofes. Sein rât an den saeldehaften Freund entfaltet mancherlei Überlegungen über Minne und huote, ritterschaft und cura domestica, die letztlich doch alle nur einem Ziel dienen:

\section{dar under lêr ich iuch wol}

iuwer êre bewarn. (2800f.)

Iwein erlistet sich die Zustimmung Laudines zu einer einjährigen Turnierfahrt, reiht sich in Artus' Gefolge ein und zieht mit dem König davon. Dem schutzlosen Land hinter ihrem Rücken schenkt keiner der Artusritter Beachtung.

Anders als Artus und die Seinen erlaube ich mir an dieser Stelle einen kurzen Blick zurück. Die Harmonie des Pfingstfestes zu Karidol ist gleich mehrfach durch übersteigertes Ehrgefühl und Konkurrenzdenken gefährdet. Der gattungstypische Einbruch der âventiure erfolgt in Gestalt von Kalogrenants maere, das von laster, schame und unêre berichtet. Iwein nimmt die Herausforderung an und gewinnt mit der Hand Laudines lant und êre. Die im Gelingen der Brunnenaventiure offenkundig gewordene Vorbildlichkeit lweins wird am Ort seiner saelde vom Artushof bestätigt. Bezogen auf die Pflichten eines Landesherrn aber bleibt das am Höchstwert der êre orientierte Ethos vollkommenen Artusrittertums ebenso defizient wie Kalogrenants âventiure Definition bei der Begegnung mit dem walttôren.

Der ungeschlachte Hirte unterscheidet sich vor allem in zwei Verhaltensweisen vom Artusritter, der seinen Wald auf der Suche nach einem passenden Gegner durchstreift. Er ist prinzipiell friedfertig. Kalogrenant atmet hörbar auf, als der mit einer riesigen Keule Bewaffnete erklärt:

\section{'swer mir niene tuot,}

der sol ouch mich ze vriunde hân.' (484f.)

Und der waltman übt, auch hierin ein Gegenbild des Artusritters, mit großer Selbstverständlichkeit ein ambet aus, indem er als meister und herre der ihm anvertrauten Herde dafür sorgt, daß die natürliche Wildheit der Tiere niemandem zum Schaden gereicht. Herrschaft, verstanden als ein Amt, das der Wahrung von Recht und Frieden dient.

Wenn es erlaubt ist, die scharf herausgearbeitete Opposition zwischen dem Artusritter Kalogrenant und dem waltôren im vorgeschlagenen Sinne zu deuten, ergibt sich die Möglichkeit, die Intention näher zu bestimmen, mit der Hartmann an sein Publikum herangetreten ist. Im Personenverbandsstaat des Mittelalters wird 
Herrschaft primär durch den Geblütsadel ausgeübt. Wer einem vornehmen Geschlecht entstammt, ist schon durch seine geburt zur Herrschaft berufen. Solange offenkundig ist, daß seine persönliche tugent den hohen Anforderungen entspricht, die seine Abkunft an ihn stellt, und solange die Heilskraft des ererbten Geblüts sichtbar im glückhaften Gelingen seiner Taten zutage tritt, bleibt seine gesellschaftliche Position unbestritten, denn er kann sich der Anerkennung seiner Standesgenossen wie auch der in der Schutzherrschaft stehenden armen liute gewiß sein. Anders ausgedrückt: der ungeschmälerte Besitz von êre 'öffentlichem Ansehen' legitimiert die Ausübung von Herrschaft.

Die Kirche, schon seit dem 10. Jahrhundert darum bemüht, ein spezifisch christliches Adeisethos auszubilden, setzte den in der Laienwelt heimischen Vorstellungen eine eigene Auffassung entgegen, in der sich der Amtsgedanke mit der Forderung nach Idoneität, der moralischen Eignung für das Amt, verband.

Hartmann steht schon im Erec erkennbar unter dem Einfluß reformkirchlichen Denkens, und sein Armer Heinrich enthält eine rigorose Auseinandersetzung mit dem Selbstverständnis des zeitgenössischen Adels. Da liegt die Vermutung nicht fern, er könne im Iwein ähnliche Ziele verfolgt haben und sein adelsstolzes Publikum mit der aus christlicher Sicht beantworteten Frage konfrontiert haben, unter welchen Bedingungen Herrschaft gerechtfertigt sei. Ob diese Annahme zutrift, kann nur ein Blick auf den zweiten Teil des Romans zeigen.

An der Seite Gaweins von Turnier zu Turnier ziehend, kehrt Iwein mit Ehren überhäuft an den Artushof zurück. Hier ergreift ihn die Sehnsucht nach Laudine, und dem Liebenden wird bewußt, daß er den ihm gesetzten Rückkehrtermin versäumt hat und an seiner Frau schuldig geworden ist. Von Schmerz und Reue überwältigt, vermag Iwein nicht mehr wahrzunehmen, was um ihn herum vorgeht. Seine Herauslösung aus der Artuswelt beginnt, noch ehe Lunete erscheint und im Auftrag Laudines die Ächtung des Wortbrüchigen fordert. Er habe im Amt des Landesherrn versagt, Laudine entehrt und es auch Lunete gegenüber an schuldiger Dankbarkeit fehlen lassen. Die öffentlich erhobene Anklage und das Bewußtsein seiner Schuld treffen Iwein zutiefst. Er verliert die Selbstachtung und flieht in die Einsamkeit. Hartmanns Wortwahl bezieht die Flucht des in seiner Existenz vernichteten Artusritters unüberhörbar auf Iweins heimlichen Aufbruch in die Brunnenaventiure zurück: er stal sich swigende dan (3227). Unbedingtes Ehrstreben führt letztlich in die Katastrophe.

Geistig umnachtet und ausschließlich darauf bedacht, die elementarsten 
Lebensbedürfnisse zu befriedigen, gleicht das Dasein des tôren in dem walde dem unvernünftiger Tiere. Aber in der Selbstentfremdung liegt auch die Chance zur Gewinnung einer neuen Identität. Hartmann deutet dies an, indem er Iweins verwildertem Äußeren Züge verleiht, die auf den anderen walttôren zurückverweisen. Iweins Reintegration in die menschliche Gesellschaft beginnt mit dem Eremiten, der inn furchtsam mit Brot und Wasser zu besänftigen trachtet. Iwein nimmt die Gaben dankbar an und lernt, Gutes mit Gutem zu vergelten - er bringt dem einsidel im Austausch Wildbret. Den Weg aus der Einöde, dem Lebensbereich des Eremiten, in die von diesseitsbejahenden Menschen bevölkerte Welt ebnen ihm drei Frauen, die den Schlafenden an einer Wundnarbe erkennen und sich seiner annehmen, weil sie auf seine Hilfe hoffen. Max Wehrli hat, ältere Beobachtungen von Willson und Hatto ausbauend, zeigen können, daß die Szenen um Iweins Entdeckung und Erwachen voller Anspielungen auf christlich-biblische Vorstellungen stecken, die dem Geschehen einen "österlichen Sinn" verleihen.

Im Buch Hiob $(20,4-8)$ und den Psalmen $(72,20)$ wird das Leben der Gottfernen mit dem Schlaf gleichgesetzt und einem flüchtigen Traum verglichen. Die gleiche Bildlichkeit scheint Paulus vorauszusetzen, wenn er im Römerbrief (13,11-12) mahnt: hora est jam nos de somno surgere [. . . ] Abjiciamus ergo opera tenebrarum, et induamur arma lucis. Wenn es erlaubt ist, auch diese Bibelstellen bei der Deutung der von Wehrli interpretierten Szenen heranzuziehen, erhalten die mit der Frage bistuz, Iwein, ode wer? einsetzenden Reflexionen des aus tiefer Umnachtung zu sich kommenden und dann auch zu sich selbst findenden Protagonisten eine weitere Bedeutungsnuance. Iweins tastende Frage hân ich geslâfen unze her?, wenig später abgewandelt erneuert ist mir getroumet mîn leben?, enthält, auf die biblische Metaphorik bezogen, die heraufdämmernde Einsicht, daß artushaftes Aventiurerittertum Gottferne bedeutet. Zu neuem Leben erwacht, wird Iwein die paulinischen Waffen des Lichts ergreifen, die traditionell auf die christlichen Tugenden gedeutet werden.

Iweins Retterin, die Herrin von Narison, wird von einem abgewiesenen Freier bedrängt, der sie mit Gewalt zur Ehe zwingen möchte. Der von den Folgen der tobesuht Geheilte leistet die erhoffte Hilfe. Sein Kampf gegen den heiratslustigen Grafen Aliers zeigt unübersehbare Parallelen zur Begegnung mit Ascalon. Auch hier gewinnt Iwein erst nach erbittertem Widerstand die Oberhand, auch hier sucht der Gegner sein Heil in der Flucht zur eigenen Burg, auch hier kann Iwein ihn erst an dem tor ergâhen (3775). Aber Aliers behält sein Leben. Iwein zwingt ihn, Schadenersatz zu 
versprechen und die allzu heftig Umworbene künftig in Frieden zu lassen. Nicht ungezügeltes Ehrstreben bestimmt sein Handeln, sondern jene Dankbarkeit, die er den hilfreichen Frauen schon an der Stätte seines Erwachens zugesagt hat:

nû vüeret mich mit iu hin:

sô handelt ir mich harte wol.

und gedienez immer als ich sol. (3634-36)

Anders als ein Artusritter, der ausgezogen ist, in der âventiure sein Glück zu machen, verzichtet Iwein auf die Hand der frouwe von Narison und auf die Herrschaft über ihr Land.

Weiterziehend hört er das wütend klagende Gebrüll eines Löwen, den er gleich darauf im Kampf mit einem feuerspeienden Drachen antrifft. Er entschließt sich, dem edelen tiere zu helfen und macht dem Drachen den Garaus. Edelkeit, nobilitas meint im Verständnis des Mittelalters den Besitz von Tugenden. Iweins Entscheidung für das edle Tier ist eine sittliche Entscheidung. Er ergreift Partei für das Gute und widersteht dem Bösen. Und es ist eine endgültige Entscheidung. Der dankbare Löwe überantwortet sich in die Obhut lweins und steht ihm bis an das Ende seines Lebens bei, weil er sich verpflichtet weiß, Gutes mit Gutem zu vergelten. Fortan ist Iwein der ritter mittem leun. Er legt seinen alten Namen ab und mit inm das alte Selbstverständnis. Isidor erläutert das Wort nobilis mit dem Satz: Nobilis, non vilis, cuius et nomen et genus scitur (Et. X, 184). Der Löwenritter beruft sich nicht auf seine Ahnen und ist auch nicht darauf aus, sein persönliches Ansehen zu mehren. Für ihn zählt nur das Ethos. So erklärt sich seine beharrliche Forderung: ich wil sin erkant bî mînem lewen (5496f.)

Die vier Aventiuren des zweiten Teils, in denen der Löwe als lweins geverte strukturell die Position einnimmt, die bisher den Artusrittern Keii und Gawein zukam, sind in einem doppelten Kursus angeordnet, dessen gleichgebaute Hälften dem Schachtelprinzip folgen. Die beiden Rahmenepisoden handeln von Gerichtskämpfen. Nach der Hilfszusage des Löwenritters ist jeweils eine eigenständige Aventiure eingeschaltet, deren retardierende Wirkung durch Einschübe erhöht ist. Auch die Geschichten, die in den beiden Einschüben erzählt werden, haben eigenes Gewicht. Endlich rundet die Wiederherstellung des Rechts das Geschehen der rahmenbildenden Episoden ab.

Die Forschungssituation und der Stand der hier vorgetragenen Überlegungen erlauben es, die eben genannten Aventiuren zunächst ganz summarisch zu behandeln. 
Lunete ist angeklagt, weil sie ihre Herrin zur Heirat mit Iwein überredet und damit Unglück über das ganze Land gebracht hat. Es ist daher nur recht und billig, daß Iwein für sie eintritt. Indem er ihre Unschuld erweist, tilgt er auch die eigene Schuld und darf fortan mit Fug und Recht behaupten, daß er die hulde Laudines ohne eigenes Verschulden entbehren muß. Die âventiure beginnt nicht umsonst am Wunderbrunnen, dem Ort der Kämpfe gegen Ascalon und Keii, und führt zur Wiederbegegnung mit Laudine, die Lunetes Retter jedoch nicht als Iwein identifizieren kann und sich erst recht unfähig zeigt, ihn bî dem lewen zu erkennen.

Auch im Kampf gegen den Riesen Harpin, der einem Schwager Gaweins und dessen Familie schweres Unrecht zugefügt hat, leistet lwein unschuldig in Not geratenen Mitmenschen die dringend benötigte Hilfe. Aventiure als Wiederherstellung verletzten Rechts, als Akt des Erbarmens und als dankbar erwiesener Freundesdienst.

Persönliche Beziehungen, wie sie Iwein mit Lunete und Gawein verbinden, spielen in den beiden folgenden Aventiuren keine Rolle. Die ältere Tochter des Grafen vom Schwarzen Dorn möchte ihre Schwester um deren rehtez erbe bringen und findet, als ein Gerichtskampf unumgänglich wird, die Unterstützung des allzu arglos einwilligenden Gawein. Ihre Schwester macht sich auf die Suche nach dem Löwenritter. Als eine Botin der unterwegs Erkrankten ihn endlich erreicht und den Wunsch äußert, er möge sich freundlich geneigt zeigen, die erbetene Hilfe zu gewähren, erhält sie zur Antwort:

'ichn habe gnâden niht:

swem mîns dienstes nôt geschiht

und swer guoter des gert,

dern wirt es niemer entwert.' (6001-04)

Im Geiste dieser programmatischen Worte kann der ritter mittem leun im Gerichtskampf mit Gawein sein Teil dazu beitragen, daß der jüngeren Grafentochter Gerechtigkeit widerfährt.

Doch zuvor muß er auf der Burg zum Schlimmen Abenteuer des wirtes gewonheit Genüge tun. Wer im Kampf mit zwei Riesen besteht, erlangt êre, lant und tohter des Burgherrn. Als Iwein auch hier mit Hilfe des Löwen den Sieg erringt, dringt der Hausherr unter Aufbietung aller ihm zur Verfügung stehenden Mittel auf die Einhaltung der costume. Aber der ritter mittem leun verweigert die Annahme des Siegespreises und zwingt den Burgherrn, dreihundert juncvrouwen freizulassen, die als seine Gefangenen entehrende Fronarbeit verrichten müssen. Die Gesetze der costume, für den Artusritter unabweisbares Gebot, haben für Iwein ihre Ver- 
bindlichkeit verloren. Maxime des Löwenrittertums ist allein der selbstlose Dienst am notleidenden Nächsten.

Hartmann hat den beiden Erzählungen, die sich als sogenannte 'Einschübe' aus der Harpinaventiure und dem Schlimmen Abenteuer entfalten, größeres Eigengewicht verliehen und so einen Hintergrund geschaffen, vor dem sich das neue Dienstethos des ritters mittem leun scharf von seinen überwundenen Vorstufen abhebt. Die Erzählung von Ginovers Raub berichtet von einem fremden Ritter, der am Artushof erscheint und vom König verlangt, er solle dem Ruf unübertroffener Freigebigkeit gerecht werden und ihm eine offene Bitte erfüllen. Artus hat Bedenken. Aber seine Ritter fürchten für die Ehre des Hofes und ringen ihm die Zustimmung ab. Der Fremde erbittet sich Ginover und reitet mit ihr davon. Die auf Ehre bedachten Tafelrunder haben den Hof in Schande gestürzt und müssen ausziehen, die Königin zu befreien, um die Schmach wieder zu beseitigen. So kommt es, daß Lunete am Artushof vergeblich Hilfe sucht. Die Sorge um die eigene Reputation hat ihn als Ort der Zuversicht für Notleidende neutralisiert.

Auch die Geschichte der dreihundert Edelfräulein, die auf der Burg zum Schlimmen Abenteuer als Textilarbeiterinnen ausgebeutet werden, demonstriert die Folgen ehrversessenen Aventiurerittertums. Schon in jungen Jahren darauf bedacht, sich einen Namen zu machen, zieht der landes herre eines Inselreiches auf Abenteuer, unterliegt im Kampf gegen die später vom Löwenritter besiegten Riesen und erkauft sich seine Freiheit mit dem Versprechen, alljährlich dreißig junge Damen als Tribut zu stellen. Statt den Schutz zu gewähren, den er ihnen als Landesherr schuldet, bürdet er den wehrlosen Frauen bedenkenlos die Folgelasten seinen Ehrstrebens auf. Wo Ehrgewinn und âventiure als Höchstwerte ritterlichen Daseins gelten, pervertiert die Schutzherrschaft zur Karikatur.

Die einzelnen Glieder der zum doppelten Kursus geordneten Episodenkette des zweiten Teils sind auch durch das Prinzip der Steigerung auf einander bezogen. Die Aventiuren der zweiten Reihe nehmen wichtige Elemente oder Motive ihrer in analoger Position stehenden Partneraventiuren aus der ersten Reihe überbietend wieder auf. Auch die Beobachtung, daß die Hilfe des Löwenritters in der ersten Aventiurengruppe Personen zugute kommt, die ihm nahestehen, gehört in diesen Zusammenhang. In der Forschung ist wiederholt darauf hingewiesen worden, daß Iwein hier noch nicht die reine triuwe verkörpere, die inn später auszeichnet. Der Gerichtskampf für Lunete und der Gawein erwiesene Freundesdienst erscheinen in dieser finalen Sicht primär als 
Status noch nicht erreichter Volikommenheit, als Zustand, den es zu überwinden gilt. Das hat seine Berechtigung. Und doch gerät auf diese Weise aus dem Blick, welche Rolle der Dankbarkeit als Motiv menschlichen Handelns im Sinngefüge des Hartmann'schen Romans zukommt.

Iwein geht den Weg in eine Krise, in der er über die Erfahrung der Selbstentfremdung zu sittlicher Erneuerung findet. Die beiden Szenen, in denen die neue Identität des gewandelten Iwein erkennbare Konturen annimmt, der Aufenthalt in Narison und die Löwenepisode, zeigen einen Menschen, den Lunetes Vorwurf, er habe Laudine das Gute, das sie ihm getan, nicht mit Gutem vergolten und sich auch ihr gegenüber undankbar gezeigt, nicht mehr zu treffen vermag. Dankbarkeit ist Iweins einziges Motiv für die Befreiungstat, mit der er seine Retterin aus der Bedrängnis durch den Grafen Aliers erlöst. Dankbarkeit bewegt den Löwen, sich für immer Iwein anzuschließen und in rehter triuwe eine Gemeinschaft zu begründen, die es gestattet, seinen Herrn bi dem lewen zu erkennen. Was den Löwenritter am Beginn seines nach dem Descensus in den Wahnsinn eingeschlagenen Weges auszeichnet und von der überwundenen Lebensform des Artusritters abhebt, ist offenbar nicht zuletzt die Bereitschaft, Dank abzustatten, zu dem er sich verpflichtet sieht. Wie erklärt es sich, daß Hartmann der Dankbarkeit eine so entscheidende Bedeutung beimißt?

Als hilfreich erweist sich überraschend die Kommentierung von Psalm 108,4-5: Pro eo ut me diligerent, detrahebant mihi: ego autem orabam. Et posuerunt adversum me mala pro bonis: et odium pro dilectione mea. Nach einer von Augustinus und Cassiodor bis zu Petrus Lombardus reichenden Tradition bildet das reddere bona pro bonis, die Bereitschaft, erwiesene Wohltaten dankbar mit Gutem zu vergelten, den ersten Schritt auf einem dreistufigen Wege, der über das non reddere malum pro malo, den Verzicht auf Vergeltung zugefügten Übels, zum reddere bonum pro malo führt, das als Ausdruck christlicher Feindesliebe verstanden wird und daher mit Cassiodor als perfectissimum virtutis genus bezeichnet werden kann.

Dem dreistufigen Weg zur vollkommenen Tugend entsprechen drei gradus, in denen - ich zitiere wieder Cassiodor - peccatum omne contrahitur 'alle Sünde vollständig versammelt ist': non reddere bona pro bonis, reddere mala pro malis und reddere mala pro bonis.

Es ist mir nicht darum zu tun, die mitgeteilte moraltheologische Auffassung, die übrigens auch in Kommentaren zu anderen Bibelstellen deutliche Spuren hinterlassen hat, zum Angelpunkt jedes /wein-Verständnisses zu erklären und etwa im Stationenweg des Protagonisten den dreistufigen ordo reddendi Augustins wiederzuentdecken. Wichtig 
ist mir nur eines: daß die bereitwillig erfültte Dankespflicht hier als erste Stufe in einem System ihren festen Platz findet, das sich ausdrücklich als Summe aller christlichen Tugenden versteht. Denn von hier aus erhält die starke Betonung der Dankbarkeit in den beiden ersten Episoden nach Iweins Erwachen einen präzisen Sinn: sie macht augenfällig, welchem Ethos der Löwenritter sich von nun an verpflichtet weiß.

Die aus dem ordo reddendi ableitbaren Kriterien erlauben es auch, Laudines Verhalten Iwein gegenüber anders und, wie ich meine, besser als bisher zu beurteilen. Doch muß ich den Beweis für diese Behauptung hier schon aus zeitlichen Gründen schuldig bleiben. Dagegen ist es im vorliegenden Zusammenhang unerläßlich, daran zu erinnern, daß die Dankbarkeit in einem offenbar weit verbreiteten, von Autoren wie Cicero, Ambrosius und Wilhelm von Conches bezeugten Verständnis als genus 'Spielart, Untertugend' der iustitia galt. Ciceros De Officiis und De Inventione, wo diese Auffassung vertreten wird (De off. I, 7, 20-23 u. I, 15, 48; De inv. II, 53, 160-61) fanden schon im Trivium als Schullektüre Verwendung. Die vielgelesene Pflichtenlehre des Ambrosius, De Officiis Ministrorum, machte Ciceros Gedanken in einer speziell für Kleriker bestimmten Fassung publik (1, cap. 31), und das Moralium Dogma Philosophorum Withelms von Conches (hier: ed. Holmberg, S. 18f. u. 23) erlebte, abgesehen von seiner überreichen handschriftlichen Überlieferung, erstaunlich früh Übersetzungen in fünf Volkssprachen.

Iustitia ist die Kardinaltugend, der seit Aristoteles die Aufgabe zufält, unter Wahrung des Gemeinwohls jedem das Seine zu schaffen. Daher gilt die Gerechtigkeit als vornehmste Herrschertugend.

Die Taten des Löwenritters dienen ausnahmslos der Abwehr von Unrecht und der Wiederherstellung gebrochenen Rechts. In diesen Zusammenhang verweist auch der Löwe. Hartmann hat der sicher publikumswirksamen Märchenfigur des täppisch dankbaren Vierbeiners in einigen Szenen komische, ja burleske Wirkungen abgewonnen. Als signum aber hat das aus den Klauen des Bösen befreite edele tier, das sich fortan in Iweins pflege begibt und inn in seinen Kämpfen unterstützt, die Bedeutungen 'Recht' und 'Gerechtigkeit' im Doppelsinn des mittellateinischen Wortes iustitia. Als eifriger Mitstreiter Iweins macht der Löwe unmittelbar sinnfällig, daß sein Herr auf der Seite des Rechts steht und verhilft ihm zum Sieg. Daß er auch die herausragende Eigenschaft des Löwenritters, seine Gerechtigkeit, meint, hat Hartmann in einer kleinen Szene modellhaft herausgearbeitet. Gaweins Schwager und die Seinen nehmen staunend wahr, daß der mächtige Löwe friedlich bei lwein liegt niuwan als ein 
ander schâf (4817) - und schließen aus der Schafsnatur des Raubtieres auf den muot ihres Gastes: er dûhtes biderbe unde guot (4812). So ist es auch zu verstehen, daß Iwein darauf beharrt, bî dem lewen erkant zu werden. Sein geverte weist ihn als vir iustus aus, und das geforderte Erkennen impliziert die Anerkennung der Einsicht, daß nicht ein glanzvoller Name zur Herrschaft qualifiziert, sondern nur das rückhaltlose Eintreten für das Recht der Notleidenden und Hilfsbedürttigen.

Als Iwein sich ein letztes Mal vom Arushof davonstiehit und, diesmal von seinem Löwen begleitet, am Wunderquell ein Unwetter auslöst, tut er es nicht, um êre zu gewinnen, sondern aus minnender nôt. Und er darf die Erfahrung machen, daß Laudines herrenloses Land des ritters mittem leun bedarf. Die Aventiuren des zweiten Teils erweisen sich durchweg als Sühneaventiuren. Nicht öffentlich erwiesenes Ansehen legitimiert zur Herrschaft, sondern vorbehaltlos geübte Gerechtigkeit.

Die hier vorgeschlagene Deutung scheint es zu ermöglichen, alle Einzelszenen und -figuren des erzähiten Geschehens auf einer Sinnebene zu integrieren und auf die gattungstypische Symbolstruktur des Doppelweges zu beziehen. Sie erlaubt es, Hartmann eine Intention zu unterstellen, die mit seinem Gesamtwerk im Einklang steht und im erschließbaren Selbstverständnis des zeitgenössischen Adels ihren außerliterarischen Bezugspunkt findet. Aber vermag sie auch den Widerspruch zwischen der Artuskritik der Romanhandlung und dem ihr vorausgehenden Artuslob aufzulösen? Ist sie überhaupt mit dem Prolog vereinbar?

Um diese Frage zu beantworten, muß man noch einmal auf die letzten Szenen zurückblicken, die am Artushof spielen, und sich die Eindrücke vergegenwärtigen, mit denen Hartmann seine Hörer von hier entläßt. Gawein und Iwein haben den Gerichtskampf um das Erbe der Grafentöchter ohne greifbaren Erfolg abgebrochen, sich zu erkennen gegeben und wetteifern nun im Bemühen, dem Freunde die Ehre des Sieges zuzusprechen. Ihre Freundschaft, von beiden ausdrücklich als verpflichtend apostrophiert, läßt dem artushaften Ehrdenken keinen Entfaltungsraum. Als Artus über ihr situationswidriges Verhalten Aufklärung wünscht, bekennt Gawein, vor einer sicheren Niederlage gestanden zu haben. Er, der sich der älteren Grafentochter bedenkenlos zur Verfügung gestellt hatte und ihre zuspätgekommene Schwester lakonisch wissen ließ waeret ir mir ê komen [. . . J iu waer mîn helfe gereit, hat gelernt, nach Recht und Unrecht zu fragen, denn er begründet sein Votum mit der Einsicht:

diu juncvrouwe hât rehtes niht 
vür die man mich hie vehten siht:

ir swester ist mit rehte hie.

sô half ouch got dem rehten ie. (7625-28)

Sein Eingeständnis ermöglicht es Artus, die Entscheidung an sich zu ziehen und die ältere Schwester mit einer Fangfrage zu überführen. Innerlich haben er und sein Hof schon längst für die Jüngere Partei ergriffen, denn diu edele und diu schoene, diu gewizzen, diu unhoene, diu süeze, diu guote, diu suoze gemuote, diu niuwan süezes kunde (7297ff.) - auch Hartmann wird nicht müde, ihre lautere Gesinnung zu preisen - hatte nicht länger verantworten wollen, daß einer der beiden Kämpfer ihretwegen seine Ehre oder das Leben verliert, und sich bereit erklärt, auf ihr Erbteil zu verzichten. Ihre selbstlose Rücksichtnahme auf andere und die Zusicherung, der Schwester nichts nachzutragen - non reddere malum pro malo - liegen weitab vom Geist der âventiure-Definition Kalogrenants. Aber sie finden am Artushof bewundernde Anerkennung und bestimmen den König, von seinem hovereht so Gebrauch zu machen, daß die halsstarrige ältere Schwester mit gewalt und vorhte in die einer Rechtsbrecherin gesetzten Schranken verwiesen wird. Damit übernimmt Artus eine Funktion, die in anderen Sühneaventiuren dem Löwen zukommt, der diesmal von Iwein unterwegs zurückgelassen worden ist. Erst als Artus Recht geschaffen hat und jeder Grafentochter das Ihre zuteil geworden ist, jagt das treue Tier auf der Fährte seines Herrn herbei. Nun ist es an Gawein, seinen Freund als den unbekannten Löwenritter zu identifizieren, der seine Schwester vom Riesen Harpin befreit hat. Der Akt des Erkennens, der damit einsetzt, bringt Gawein zunächst die bestürzende Einsicht, den mit dem Schwert in der Hand bekämpft zu haben, der mehr als andere auf seine Dankbarkeit Anspruch gehabt hätte:

$$
\begin{aligned}
& \text { ich mac wol iemer sîn unvrô, } \\
& \text { daz ich iu sus gedanket hân } \\
& \text { des ir mir guotes hânt getân. } \\
& \text { den risen sluogent ir durch mich.(7746-49) }
\end{aligned}
$$

Aber es bleibt nicht bei nachdenklicher Betroffenheit. Angesichts des Löwen erkennt Gawein, welche Bedeutung Recht und Gerechtigkeit im Leben seines Freundes erlangt haben, und vermag sich dem Anspruch der vom ritter mittem leun bezeugten Wahrheit nicht länger zu entziehen. Gerechtigkeit üben, das heißt für ihn in der konkreten Situation vor allem, seiner Dankespflicht genügen:

unde esn letze mich der tôt, ich gedienez iemer als ich sol. 
ich erkenne iuch bî dem lewen wol. (7760-62)

Das als Lebensmaxime formulierte Bekenntnis Gaweins zur sittlichen Pflicht, Gutes stets mit Gutem zu vergelten, verweist auf die spontane und doch lebenslange Dankbarkeit des Löwen zurück und wiederholt iweins Worte an das hilfreiche Edelfräulein der Dame von Narison: ich gedienez iemer als ich sol. Das kann nur eines besagen. Der Musterritter des Artushofes steht am Beginn des Weges, den Iwein nach dem Erwachen aus dem Wahnsinn eingeschlagen hat, und ist entschlossen, seinem Freunde nachzueifern. Auch der Artushof hat sich am Löwenritter ein bilde genommen. Von dem König aber, der einem solchen Hof vorsteht, kann Hartmanns Prolog mit Fug und Recht sagen:

$$
\begin{aligned}
& \text { Swer an rehte güete } \\
& \text { wendet sîn gemüete, } \\
& \text { dem volget saelde und êre. } \\
& \text { des gît gewisse lêre } \\
& \text { künec Artûs der guote [. . . ] }
\end{aligned}
$$

Zitate nach: Iwein. Eine Erzählung von Hartmann von Aue. Herausgegeben von G. F. Benecke und K. Lachmann. Neu bearbeitet von Ludwig Wolff. Siebente Ausgabe Band 1: Text. Berlin 1968.

Die Vortragsform der vorstehenden Ausführungen wurde unverändert beibehalten. Das schließt den Verzicht auf eine nachträgliche Ergänzung von Anmerkungen ein. Die Auseinandersetzung mit der Forschung mußte schon aus Raumgründen einer geplanten späteren Publikation vorbehalten bleiben. 